\title{
Trauma, Posttraumatische Belastungsstörung und Substanzabhängigkeit: Eine Literaturübersicht
}

\author{
Trauma, Posttraumatic Stress Disorder and Substance Abuse: \\ A Review of the Literature
}

\section{Zusammenfassung}

In repräsentativen Bevölkerungserhebungen und in klinischen Untersuchungen konnte ein Zusammenhang zwischen einer Alkohol- und Drogenabhängigkeit und dem Vorliegen eines traumatischen Ereignisses in der Vergangenheit der Patienten bzw. einer aktuellen Posttraumatischen Belastungsstörung sehr häufig belegt werden. Die folgende Literaturübersicht gibt einerseits einen Einblick in den Forschungsstand mit einem Fokus auf Gewalterfahrungen in Kindheit und Jugend und diskutiert andererseits mögliche kausale Zusammenhänge zwischen traumatischen Ereignissen und der Entwicklung einer Substanzabhängigkeit.

\section{Schliusselwörter}

Abhängigkeit · Sucht · Trauma · Posttraumatische Belastungsstörung · kindliche Gewalterfahrungen

\section{Abstract}

In representative population surveys and clinical trials there is often a correlation between substance abuse and a history of trauma and a lifetime prevalence of posttraumatic stress disorder in the patients. The following review of the literature will give on one side an overview regarding actual studies, with a focus on child sexual and physical abuse, and on the other side discuss potential causal relationships between trauma and addiction.

\section{Key words}

Dependence · addiction - trauma - posttraumatic stress disorder · childhood sexual and physical abuse

\section{Einleitung}

Der Einstieg in den Konsum legaler und illegaler Drogen hat viele denkbare Zugänge: u.a. Neugier, die Suche nach einem Ausweg aus belastenden Problemen, Verstärkung positiver Erlebnisse und die Verfügbarkeit der Substanzen. Eine Phase des Ausprobierens auch illegaler Substanzen begleitet den Prozess des Ablösens vom Elternhaus. Das Abgleiten in die Abhängigkeit mit ihren mannigfaltigen Facetten persönlichen Leids ist wiederum bedingt durch vielfältige Einflussgrößen, nicht zuletzt durch das Abhängigkeitspotenzial der Substanzen selbst.
Bei der Diskussion um die Ätiologie der Suchtmittelabhängigkeit richtet sich in der letzten Zeit der Fokus verstärkt auf den Bereich der Realtraumatisierungen. Traumatisierungen als Risikofaktoren der Entwicklung einer Abhängigkeitserkrankung oder anderer psychiatrischer Erkrankungen sind mittlerweile gut belegt, wenn auch der Zusammenhang noch einer Klärung bedarf. Nicht alle Menschen mit traumatischen Erfahrungen werden substanzabhängig oder erkranken an psychischen Erkrankungen und nicht alle Abhängigkeitskranke berichten über traumatische Erfahrungen vor der Manifestation ihrer Sucht. Der Anteil der Suchtmittelabhängigen mit traumatischen Erlebnissen in ihrer

Institutsangaben

Institut für Interdisziplinäre Suchtforschung, Universitätsklinikum Hamburg-Eppendorf

Korrespondenzadresse

Dr. Silke Kuhn · Zentrum für Interdisziplinäre Suchtforschung, Universitätsklinikum Hamburg-Eppendorf .

Martinistraße 52 ·20246 Hamburg·E-mail: skuhn@uke.uni-hamburg.de

Bibliografie

Suchttherapie 2004; 5: 110 - 117 (c) Georg Thieme Verlag KG Stuttgart · New York

DOI 10.1055/s-2004-813526

ISSN 1439-9903 
Lebensgeschichte und mit Symptomen einer Posttraumatischen Belastungsstörung oder anderen psychischen Beschwerden, die als Traumafolgen angesehen werden können, ist jedoch sehr hoch. Die Entwicklung neuer integrativer therapeutischer Behandlungskonzepte, die gleichzeitig sowohl sucht- als auch traumaspezifische Aspekte enthalten, zeigt deutlich, dass diese Abhängigkeitskranken zunehmend in Behandlungseinrichtungen wahrgenommen werden.

Dabei darf nicht vergessen werden, dass es sich bei Patienten mit traumatischen Erfahrungen, die gleichzeitig auch suchtmittelabhängig sind, um eine spezielle Gruppe von Personen handelt, nämlich um diejenigen, die sich nicht aufgrund der Traumafolgen suizidiert haben. Gleichwohl werden auch nicht diejenigen Personen erfasst, die trotz traumatischer Erlebnisse weder eine Suchterkrankung noch andere psychische Störungen entwickelt haben.

In der Auseinandersetzung mit der Literatur fällt auf, dass es sich bei den Autorinnen und Autoren, im Gegensatz zur gängigen wissenschaftlichen Praxis, vorwiegend um weibliche Autoren handelt. Dabei bleibt es ungeklärt, ob Frauen vorrangig dieses Forschungsfeld besetzt haben, z.B. über die Beschäftigung mit sexuellem Missbrauch an Mädchen und Frauen, oder ob Männer diesen Bereich der Forschung eher meiden.

\section{Begriffsklärung}

\section{Trauma}

Das Wort Trauma kommt aus dem Griechischen und bedeutet „Wut“ oder „Verletzung“ und meint „seelische Verwundung“. Ein Trauma bedeutet auf jeden Fall eine massive Unterbrechung der Lebensgeschichte. Der Begriff Trauma wird in der Umgangssprache teilweise inflationär benutzt, erfährt jedoch im klinischen und wissenschaftlichen Sprachgebrauch eine klare und engere Definition.

Ein traumatisches Ereignis ist definiert als die Erfahrung von Gewalt, die das eigene Leben oder die körperliche Integrität bedroht und starke Angst, Schrecken und Hilflosigkeit auslöst. Auch das Mitansehen von Gewalt gegen andere fällt unter diese Definition. Die Internationale Klassifikation psychischer Störungen (ICD-10) definiert ein Trauma als ein Ereignis oder eine Situation außergewöhnlicher Bedrohung, die bei fast jedem eine tiefe Verzweiflung hervorrufen würde. Das DSM-IV gibt dagegen wesentlich klarere und engere Leitlinien vor und beschreibt als Ergebnis eine intensive Angst, Hilflosigkeit oder Entsetzen. $\mathrm{Zu}$ den am häufigsten untersuchten traumatischen Erfahrungen gehören Reaktionen auf Kriegserlebnisse, Naturkatastrophen, sexuelle Übergriffe und das Erleben von körperlicher Gewalt.

Das Spektrum von Folgen, die das Erleben eines Traumas bewirken kann, ist sehr vielfältig und abhängig von der Art, Dauer und Intensität des erlebten Traumas, wobei lang andauernde negative Folgen nicht zwingend eintreten müssen. Vielfältige psychische und psychosomatische Auswirkungen traumatischer Ereignisse sind denkbar, worunter die Posttraumatische Belastungsstörung (PTBS) mit ihrem direkten Bezug zu einem erlebten Trauma sehr häufig untersucht wurde (s. [1-6]).

\section{Posttraumatische Belastungsstörung (PTBS)}

Die Posttraumatische Belastungsstörung (PTBS) oder Posttraumatic Stress Disorder (PTSD) ist eine in den internationalen Klassifikationssystemen ICD-10 und DSM IV detailliert beschriebene Störung. Sie ist die einzige Erkrankung mit eindeutiger Ätiologie, nämlich einem der Störung zugrunde liegenden Trauma. Das erlebte Trauma muss durch ständiges Wiedererleben z. B. in Alpträumen, Flash-backs oder sich aufdrängenden Gedanken präsent sein und sich in Symptomen eines Meidungsverhalten oder erhöhter Erregbarkeit manifestieren. Die Symptome einer PTBS werden in Tab. 1 kurz dargestellt. Dabei sollte die Symptomatik länger als einen Monat andauern.

Tab. 1 Symptome einer PTBS nach ICD-10 und DSM-IV

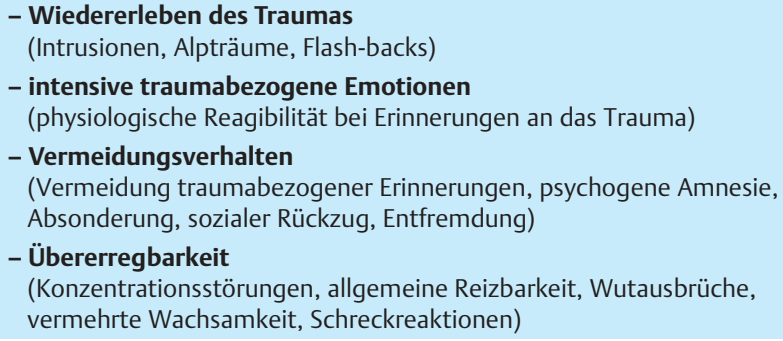

Zusammenhang zwischen Trauma und Posttraumatischer Belastungsstörung

Die Prävalenzen der der PTBS zugrunde liegenden traumatischen Ereignissen sind in dem Ausmaß ihres Vorkommens relativ uneinheitlich (s. Tab. 2). Durchweg berichten Männer über eine größere Anzahl an traumatischen Ereignissen, während Frauen auf die ihnen widerfahrenden Traumata mit einer höheren Wahrscheinlichkeit für das Entstehen einer PTBS reagieren. Frauen geben mehr Vergewaltigungen, sexuelle Belästigungen, Vernachlässigung und körperliche Gewalt in der Kindheit an, während Männer mehr körperliche Angriffe, Bedrohungen mit Waffen, Gefangenschaft oder Kriegserfahrungen berichten [7-9].

Traumatische Ereignisse sind keine seltenen Ereignisse. Ihre Prävalenzraten liegen zwischen 39\% [10] und über $80 \%$ [11]. Diese unterschiedlichen Prävalenzzahlen haben ihre Begründung, neben unterschiedlichen Stichproben, auch in der Art der Erhebung der traumatischen Ereignisse. Die sehr niedrigen Prävalenzraten für PTBS in den frühen Epidemiologic-Catchment-Area-(ECA-)Studien werden darauf zurückgeführt, dass dort nicht, wie in den weiteren epidemiologischen Studien üblich, nachdrücklich Ereigniskategorien traumatischer Erlebnisse abgefragt wurden [11].

Allein der Vergleich der Prävalenzzahlen für Traumata und PTBS belegt deutlich, dass dazwischen keine zwingende Verknüpfung besteht. So drängt sich die Frage auf, ob es traumatische Erlebnisse gibt, die mit einer höheren Wahrscheinlichkeit zur Ausbildung des Krankheitsbildes einer PTBS führen als andere. Die repräsentative Umfrage von Kessler (1995) gibt dazu wichtige Hinweise [9]. Vergewaltigung ist in dieser Studie sowohl bei 


\begin{tabular}{|c|c|c|c|c|c|c|c|}
\hline Studie & Land & $n$ & $\begin{array}{l}\text { Trauma } \\
\text { (\%) } \\
\text { Frauen }\end{array}$ & $\begin{array}{l}\text { Trauma } \\
\text { (\%) } \\
\text { Männer }\end{array}$ & PTBS (\%) & $\begin{array}{l}\text { PTBS (\%) } \\
\text { Frauen }\end{array}$ & $\begin{array}{l}\text { PTBS } \\
\text { (\%) } \\
\text { Männer }\end{array}$ \\
\hline Helzer et al. (1987) [12] ${ }^{1}$ & USA (St. Louis) & 2493 & & & 1 & 1,3 & 0,5 \\
\hline Davidson et al. (1991) [13] ${ }^{1}$ & USA (North Carolina) & 2985 & & & 1,3 & & \\
\hline $\begin{array}{l}\text { Kessler et al. (1995) [9] } \\
\text { Kessler et al. (1999) [14] }\end{array}$ & $\begin{array}{l}\text { USA } \\
\text { 12-Monats-Prävalenz }\end{array}$ & 8098 & 51,2 & 60,7 & $\begin{array}{l}7,8 \\
3,9\end{array}$ & 10,4 & 5,0 \\
\hline Resnick et al. (1993) [15] ${ }^{2}$ & USA & 4008 & 68,9 & & & 12,3 & \\
\hline Breslau et al. (1991) [10] ${ }^{3}$ & USA & 1007 & 36,7 & 43,0 & 9,2 & 11,3 & 6,0 \\
\hline Creamer (2001) [8] & $\begin{array}{l}\text { Australien } \\
\text { Trauma: Lebenszeitprävalenz } \\
\text { PTBS: 12-Monats-Prävalenz }\end{array}$ & 10641 & 49,5 & 64,5 & 1,33 & & \\
\hline Perkonigg et al. (2000) [16] & Deutschland & 3021 & 17,7 & 25,2 & 1,3 & 2,2 & 0,4 \\
\hline Stein et al. (1997) [11] & $\begin{array}{l}\text { Kanada } \\
\text { PTBS: 1-Monats-Prävalenz }\end{array}$ & 1002 & 74,2 & 81,3 & & 2,7 & 1,2 \\
\hline
\end{tabular}

Tab. 2 Lebenszeitprävalenzen traumatischer Ereignisse und Postraumatischer Belastungsstörungen in epidemiologischen Studien

\footnotetext{
${ }^{1}$ Epidemiologische-Catchment-Area-(ECA-) Studien

2 Telefonumfrage nur Frauen

3 junge Erwachsene zwischen 21 und 30 Jahren

4 Jugendliche und junge Erwachsene zwischen 14 und 24 Jahren
}

Frauen als auch bei Männern dasjenige Ereignis, welches mit der höchsten Wahrscheinlichkeit zur Entwicklung einer PTBS führt, auch wenn es von Männern seltener berichtet wird. 65 Prozent der Männer und 45,9\% der Frauen, die Vergewaltigung als das belastendste traumatische Ereignis nannten, litten unter Symptomen einer PTBS.

Zusammenhang zwischen Traumaerleben, PTBS und Entwicklung einer Substanzabhängigkeit

Der Zusammenhang zwischen dem Erleben traumatischer Ereignisse, der Entwicklung einer PTBS und/oder Substanzabhängigkeit sowie weiterer komorbider Störungen ist durch eine Vielzahl von Studien belegt. Bei den Personen mit PTBS ist die Wahrscheinlichkeit einer Substanzabhängigkeit 2- bis 12-mal höher als in der Gruppe der Personen ohne PTBS [8-10,12]. Die Übersichtsarbeit von Brady (1997) zeigt bei sich in Behandlung befindenden Substanzabhängigen eine komorbide PTBS von 25 bis 58\% [3]. Hohe Prävalenzraten von PTBS können nicht nur durch das spezielle Untersuchungssample bedingt sein, sondern auch Ausdruck einer Retraumatisierung unter Alkohol und Drogen, die bewirkt, dass betroffenen Personen immer weniger Copingmechanismen zur Verfügung stehen, um mit den emotionalen Belastungen umgehen zu können [17].

Die zeitliche Abfolge von traumatischen Ereignissen bzw. dem Auftreten von Symptomen einer PTBS und der Entwicklung einer Substanzabhängigkeit kann nicht eindeutig beantwortet werden. Es scheinen zwei grundsätzliche zeitliche Beziehungen denkbar:

1. Trauma und PTBS liegen zeitlich vor dem Beginn der Substanzabhängigkeit.

2. Trauma und PTBS liegen zeitlich nach dem Beginn der Substanzabhängigkeit.

1) Die epidemiologische Studie von Perkonigg (2000) gibt Auskunft über die zeitlichen Zusammenhänge von Trauma/PTBS und Substanzabhängigkeit bei Jugendlichen und jungen Erwachsenen [16]. Der Beginn einer Alkoholabhängigkeit oder eines Miss- brauchs lag in 55,5\% der Fälle und der einer Drogenabhängigkeit bzw. eines Missbrauch, in 75\% der Fälle nach dem Beginn der PTBS. Die Substanzabhängigkeit erscheint demnach in einem hohen Maße sekundär. Breslau (2001) konnte einen Zusammenhang zwischen PTBS und Medikamentenabhängigkeit feststellen, jedoch keinen Zusammenhang zwischen traumatischen Ereignissen und Substanzabhängigkeit. Aufgrund ihrer Datenlage postulierte sie die Substanzabhängigkeit als das Resultat des Versuchs, die Symptome der PTBS zu lindern, und präferiert offensiv die so genannte „Selbstmedikationshypothese“ $[5,18]$. Bei Vorliegen einer PTBS ist das Risiko einer Suchterkrankung oder anderer komorbider Störungen um 4,5-mal höher als ohne, dies gilt jedoch nicht bei Traumata ohne nachfolgende Ausprägung einer PTBS (vgl. [6]).

2) Cottler et al. (1992) unterzogen die Daten der St.-Louis-Catchment-Area-Studie [12] einer spezifischen Analyse und fanden Evidenz für die zweite mögliche Annahme [19]. Der erste Drogenkonsum ging dem Beginn der PTBS voraus. Als Prädiktoren einer PTBS konnten sie unter Kontrolle möglicher weiterer Einflussgrößen Kokain- und/oder Opiatgebrauch und das Geschlecht (weiblich) ermitteln, während alleiniger Marihuanagebrauch einen, wenn auch nicht signifikanten, „protektiven“ Faktor darstellte [19]. In einer weiteren Studie an i.v. konsumierenden Drogenabhängigen fanden sie ihre vorherigen Ergebnisse bestätigt. Der Beginn des Drogengebrauchs lag vor dem PTBS auslösenden Ereignis [20]. Kritisch ist anzumerken, dass lediglich der Beginn des Konsums, nicht jedoch der Beginn einer Abhängigkeit für die Berechnungen verwendet wurde und diese Arbeiten die einzigen sind, die gegen die „Selbstmedikationshypothese“ sprechen.

\section{Zusammenhang zwischen Missbrauch und Misshandlungen sowie weiteren belastenden Faktoren in Kindheit und Jugend und späterer Substanzabhängigkeit}

Traumata in der Kindheit und Jugendzeit gelten als mögliche bahnende Ereignisse für einen späteren Gebrauch psychotroper Substanzen. Unter diesen Traumata werden vor allem sexueller Missbrauch sowie körperliche und seelische Misshandlungen verstanden. Bei der Bewertung der Prävalenzzahlen für Missbrauch in Kindheit und Jugendzeit müssen die verschiedenen Definitionen der Ereignisse und unterschiedliche Erhebungsmethoden berück- 
sichtigt werden. In den weitaus meisten Studien wurden die Folgen sexuellen Missbrauchs und/oder körperlicher Misshandlungen untersucht, seltener jedoch, weil weniger valide zu erfassen, emotionale Misshandlung und Vernachlässigung.

Die Prävalenzraten für kindlichen Missbrauch und Misshandlungen in der Gesamtbevölkerung können aufgrund uneinheitlicher Definitionen und des Schweigegebots der Täter nur ungenau sein. Internationale Schätzungen gehen davon aus, dass jedes vierte bis fünfte Mädchen und jeder zwölfte Junge bis zum Alter von 16 Jahren sexuell missbraucht werden [21 - 24].

\section{Begriffsklärung: sexueller Missbrauch und körperliche Misshandlungen in der Kindheit}

Die Ereignisse, die unter dem Begriff des sexuellen Missbrauchs subsumiert werden, reichen von obszöner Rede oder Gesten bis hin zur vollzogenen Penetration bei Kindern und Jugendlichen unter 16 Jahren bzw. 18 Jahren. Der in vielen Studien geforderte Altersunterschied von mindestens 5 Jahren zwischen Opfer und Täter ist problematisch, da oftmals Gleichaltrige oder wenig Ältere einen Teil der Täterschaft ausmachen [22, 25 -27]. Bedeutsamer scheint das Wesen der Beziehung von Täter und Opfer zu sein. Das Macht- oder Vertrauensverhältnis wird vom Täter ausgenutzt, um ein Kind oder einen Jugendlichen zur Befriedigung der eigenen sexuellen Befürfnisse zu benutzen. Das Opfer ist nicht in der Lage, sich entgegen dem vorherrschenden Machtgefälle eigenverantwortlich entscheiden zu können. Dabei ist es unerheblich, ob vom Täter Gewalt oder Zwang angewendet wird. Im Hinblick auf körperliche Misshandlungen ist es nicht einfach, zwischen durchaus immer noch üblichen körperlichen Disziplinierungsmaßnahmen und Misshandlungen zu unterscheiden. In den meisten Studien wird aber übereinstimmend von einem gewaltsamen Akt ausgegangen, der beim Kind oder Jugendlichen zu körperlichen Verletzungen führt, z. B. Schläge, Tritte, Verbrennungen etc.

\section{Studien zu kindlichen Traumatisierungen und Substanzabhängigkeit}

Die Vergleichbarkeit der Studien ist durch unterschiedliche Missbrauchsdefinitionen und ausgewählte Untersuchungssamples problematisch. Einschränkend muss auch angemerkt werden, dass es nur wenige Studien gibt, die explizit Drogenabhängige untersuchen. Zudem gibt es nur wenige Studien an Männern [28] und auch Studien, die explizit die Auswirkungen körperlicher Misshandlungen untersuchen, sind in der Minderzahl. An dieser Stelle werden Ergebnisse zweier unterschiedlicher Studienkonzeptionen vorgestellt: Studien an repräsentativen Bevölkerungsstichproben und klinische Studien an Patientinnen und Patienten mit Abhängigkeitserkrankungen.

\section{Repräsentative Studien in der Allgemeinbevölkerung}

Die Studien an der Allgemeinbevölkerung an großen Stichproben (s. Tab. 3) belegen einen grundsätzlichen Zusammenhang zwischen sexuellem Missbrauch und körperlichen Gewalterfahrungen in Kindheit und Jugend und späterer Substanzabhängigkeit [29-36].

Die Ergebnisse der ECA-Studie ergaben z. B. für beide Geschlechter bei sexuellem Missbrauch in der Kindheit ein 2,3- bis 2,5fach höheres Risiko, an einer Suchtmittelabhängigkeit zu erkranken
[29]. Die Analyse der Frauen zeigte, dass vor allem die Kombination von sexueller und körperlicher Gewalt das Risiko erhöht, eine Posttraumatische Belastungsstörung zu entwickeln [37]. Darüber hinaus gibt es einen deutlichen Zusammenhang zwischen der Schwere des sexuellen Missbrauchs und dem Auftreten psychischer Störungen im Erwachsenenalter [30, 33, 38].

Viele Studien zeigen nicht nur einen Zusammenhang zwischen sexuellem Missbrauch und körperlichen Misshandlungen in der Kindheit und einer PTBS, sondern ebenso einen zum Teil noch deutlicheren Zusammenhang mit anderen psychischen Störungen, wie Depressionen und Angststörungen (Phobien, Panikattacken) $[29-33,35,37]$.

Diese Studien belegen zudem sehr ausdrücklich das um ein Vielfaches erhöhte Suizidrisiko von Abhängigkeitskranken mit kindlicher Traumatisierung. Frauen, die in ihrer Kindheit sexuell missbraucht wurden, hatten in der Untersuchung von Muller et al. [30] ein um das 20fache erhöhtes Suizidrisiko, welches bei vollzogener Penetration auf das 70 fache anstieg [30, 39].

Das Risiko für eine spätere Drogenabhängigkeit ist bei allen Studien grundsätzlich höher als für eine Alkoholabhängigkeit, vor allem bei sexuellem Missbrauch [31, 33].

Sexueller Missbrauch oder körperliche Misshandlungen sind keine isolierten Ereignisse, sondern geschehen in der Regel auf dem Hintergrund weiterer belastender Kindheitsereignisse. Der Frage, welchen Einfluss multiple Kindheitstraumata (u.a. sexueller und körperlicher Missbrauch, körperliche Misshandlungen, Substanzgebrauch der Eltern, allgemeines Klima von Gewalt in der Familie) auf die spätere Entwicklung und die Ausbildung einer Substanzabhängigkeit und weiterer psychischer, aber auch somatischer Erkrankungen haben, ging die repräsentative ACE-Studie nach [39-42]. Die Ergebnisse sprechen für ein klares DosisWirkungs-Verhältnis. Je mehr belastende Kindheitsereignisse erfahren wurden, desto höher war das Risiko für psychische Erkrankungen im Erwachsenenalter und dies in Bezug auf eine spätere Alkoholabhängigkeit unabhängig davon, ob eine elterliche Suchterkrankung vorlag. Allerdings bildeten diejenigen, deren Eltern selbst alkoholkrank waren, eine Untergruppe mit einem sehr großen Risiko, selbst alkoholabhängig zu werden.

\section{Klinische Studien}

Der Zusammenhang zwischen kindlicher Traumatisierung und späterem Substanzkonsum konnte durch viele klinische Studien belegt werden. In ihrer Übersichtsarbeit über die Studien der letzten 30 Jahre geben Simpson \& Miller (2002) u.a. Studien an, die sich mit Traumatisierung bei Suchtmittelabhängigen beschäftigt haben [27]. Sie differenzieren jedoch nicht nach Alkohol- und Drogenpatienten. Die 47 Studien, die Aussagen über die Prävalenzraten bei erwachsenen weiblichen Suchtpatienten treffen, gehen von Prävalenzraten zwischen 9,6 und 83\% für sexuellen Missbrauch und zwischen 19 und 67\% für körperliche Misshandlungen aus. Insgesamt 22 Studien, die auch Aussagen für männliche Suchtpatienten zulassen, fanden Prävalenzraten zwischen 0,9 und 54,5\% für sexuellen Missbrauch und zwischen 7 und 90\% für körperliche Misshandlungen. Auch wenn diese Studien sich hinsichtlich ihrer methodischen Qualität sehr stark unterscheiden, macht die enorme Spannweite der prozentualen 
Tab. 3 Repräsentative Bevölkerungsstudien zum Zusammenhang von kindlichen Gewalterfahrungen und Substanzmittelabhängigkeit

\begin{tabular}{|c|c|c|}
\hline Studie & Land & $\begin{array}{l}\text { Stichproben- } \\
\text { größe }\end{array}$ \\
\hline Burnam et al. (1988) [29] & $\begin{array}{l}\text { Epidemiologic-Catchment- } \\
\text { Area-(ECA-)Studie } \\
\text { USA, Los Angeles }\end{array}$ & $\begin{array}{l}3132 \\
\text { Erwachsene }\end{array}$ \\
\hline Winfried et al. (1990) [37] & $\begin{array}{l}\text { Epidemiologic Catchment } \\
\text { Area (ECA)-Studie } \\
\text { USA, North Carolina }\end{array}$ & $\begin{array}{l}1157 \\
\text { Frauen }\end{array}$ \\
\hline Mullen et al. (1993) [30] & Neuseeland & $\begin{array}{l}1376 \\
\text { Frauen }\end{array}$ \\
\hline Duncan et al. (1996) [35] & USA, Columbia & $\begin{array}{l}4008 \\
\text { Frauen }\end{array}$ \\
\hline Wilsnack et al. (1997) [31] & USA & $\begin{array}{l}1099 \\
\text { Frauen }\end{array}$ \\
\hline Spak et al. (1997) [36] & Schweden & $\begin{array}{l}316 \\
\text { Frauen }\end{array}$ \\
\hline $\begin{array}{l}\text { Kendler et al. (2000) [33] } \\
\text { Bulik et al. (2001) [38] }\end{array}$ & USA & $\begin{array}{l}1411 \\
\text { weibliche } \\
\text { Zwillinge }\end{array}$ \\
\hline MacMillan et al. (2001) [32] & Kanada & $\begin{array}{l}7016 \\
\text { Erwachsene }\end{array}$ \\
\hline $\begin{array}{l}\text { Felitti et al. (1998) [39] } \\
\text { Edwards et al. (2003) [42] } \\
\text { Dube et al. (2003) [41] }\end{array}$ & $\begin{array}{l}\text { Adverse-Childhood-Experi- } \\
\text { ences-(ACE-)Studie } \\
\text { USA, 1. Untersuchungsgruppe }\end{array}$ & $\begin{array}{l}9508 \\
\text { Erwachsene }\end{array}$ \\
\hline Dube et al. (2002) [40] & $\begin{array}{l}\text { Adverse-Childhood-Experi- } \\
\text { ences-(ACE-)Studie } \\
\text { USA, 1.und 2. Unter- } \\
\text { suchungsgruppe }\end{array}$ & $\begin{array}{l}17337 \\
\text { Erwachsene }\end{array}$ \\
\hline
\end{tabular}

Tab. 4 Ausgewählte klinische Studien zum Zusammenhang von kindlichen Gewalterfahrungen und Substanzmittelabhängigkeit

\begin{tabular}{|c|c|}
\hline Studie & Stichproben \\
\hline Wallen \& Berman (1992) [47] & $\begin{array}{l}217 \text { stationäre Behandlung, Alk. } \\
+ \text { Drogen, } w+m\end{array}$ \\
\hline Gil-Rivas et al. (1996) [48] & 182 w + 148 m, Drogenabhängige \\
\hline Deykin \& Buka (1997) [17] & $\begin{array}{l}297 \text { Jugendliche, } 15 \text { - } 19 \text { Jahre, Alk. } \\
\text { + Drogen }\end{array}$ \\
\hline Clark et al. (1997) [49] & $\begin{array}{l}132 \text { Alkohol, } 73 \text { Kontrollen, } 14-18 \\
\text { Jahre, } w+m\end{array}$ \\
\hline Schäfer et al. (2000) [26] & 100 Polytoxikomane, $w+m$ \\
\hline Back et al. (2000) [7] & 91 Kokainabhängige, $w+m$ \\
\hline Clark et al. (2001) [50] & $\begin{array}{l}150 \text { Opiatpatienten im Methadon- } \\
\text { behandlung, } w+m\end{array}$ \\
\hline Ballon et al. (2001) [51] & $\begin{array}{l}287 \text { Jugendliche u. Erwachsene, } \\
14-24 \text { Jahre, Alk. + Drogen }\end{array}$ \\
\hline Cottler et al. (2001) [20] & 464 Drogenabhängige, $w+m$ \\
\hline Langeland et al. (2002) [52] & $122 \mathrm{~m}$ und $33 \mathrm{w}$, Alk. + Drogen \\
\hline Thompson Fullilove et al. (1993) [53] & 105 Frauen, Drogenabhängige \\
\hline Brady et al. (1994) [54] & 55 Frauen, Alk. + Drogen \\
\hline Simpson (2002) [55] & 72 Frauen, Drogenabhängige \\
\hline Simons et al. (2003) [56] & $\begin{array}{l}112 \text { Frauen in ambulanter Behand- } \\
\text { lung, Alk. + Drogen }\end{array}$ \\
\hline Triffleman et al. (1995) [57] & 46 Männer, Alk. + Drogen \\
\hline Harrison et al. (1990) [58] & $\begin{array}{l}1227 \text { männliche Jugendliche, Alk. } \\
\text { + Drogen }\end{array}$ \\
\hline w = weiblich, $m$ = männlich & \\
\hline
\end{tabular}


haben [51]. Auch berichten diese Jugendlichen und jungen Erwachsenen signifikant häufiger, dass sie Substanzen als eine Art Copingstrategie einsetzen, um dadurch besser ihre Wut beherrschen zu können. Fraglich bleibt dabei, inwieweit es sich bei beschriebenen aggressiven Impulsen nicht auch um Entzugssymptomatik handeln könnte. Simpson (2002) überprüft die häufig geäußerte Annahme, dass kindlicher sexueller Missbrauch ein Risikofaktor in der Behandlung von Suchtmittelabhängigen ist [55]. Überraschenderweise stellte sie fest, dass die untersuchten Patientinnen mit sexuellem kindlichen Missbrauch nicht häufiger wegen ihrer Suchtproblematik in Behandlung waren, jedoch häufiger wegen anderer psychischer Probleme eine psychiatrische Behandlung in Anspruch nahmen. Tendenzen der Reviktimisierung finden sich unabhängig vom Geschlecht vor allem in der Gruppe der Suchtmittelabhängigen mit schweren Missbrauchs- und Misshandlungserfahrungen [26]. Ruggiero et al. (1999) verbinden in ihrer Studie an 200 Männern unterschiedliche Missbrauchsformen in der Kindheit (sexueller Missbauch, körperliche Misshandlungen und Vernachlässigung) mit Persönlichkeitsstörungen [63]. Es zeigte sich, wie wichtig es ist, auch den Schweregrad der Gewalterfahrungen zu untersuchen, da nur die Patienten mit schweren Missbrauchs-, Misshandlungsund Vernachlässigungserfahrungen eine höhere Beeinträchtigung durch Persönlichkeitsstörungen als die Patienten ohne oder mit mäßig schweren Kindheitstraumata zeigten.

Diese frühen Erfahrungen scheinen insgesamt die Vulnerabilität für Substanzkonsum und psychiatrische Erkrankungen zu erhöhen. Der Gebrauch von Alkohol und illegalen Drogen kann als ein Versuch angesehen werden, Ängste und Depression zu reduzieren und allgemein ein niedriges Selbstwertgefühl zu stärken, Gedanken und Gefühle bez. des Missbrauchs zu blockieren oder überhaupt Empfinden zuzulassen und spürbar und erlebbar zu machen. Insofern hat der Gebrauch der illegalen Substanzen eine wichtige Funktion, auch wenn er langfristig als misslungene Copingstrategie anzusehen ist, zumal dadurch häufig ein Zirkel an belastenden Lebensumständen mit Gewalterfahrungen und Substanzabhängigkeit sowohl im weiteren Lebenslauf der Personen als auch durch die Weitergabe an die nächste Generation entsteht [40].

\section{Diskussion}

Eine große Anzahl von klinischen Studien und auch die vorliegenden Bevölkerungsstudien zeigen einen Zusammenhang zwischen traumatischen Erlebnissen, insbesondere Gewalterfahrungen in der Kindheit und späterem Alkohol- und Drogenkonsum. Es entspricht der klinischen Erfahrung, dass sexueller Missbrauch oder körperliche Misshandlungen in der Kindheit die Opfer bis ins Erwachsenenalter hinein traumatisieren können. Aufgrund der Relevanz dieser Form traumatischer Ereignisse für den Beratungs- und Behandlungsalltag in Suchthilfeeinrichtungen wird in der Diskussion vorwiegend darauf Bezug genommen.

Der Fokus bei der Beschäftigung mit Realtraumatisierungen in den vergangenen Jahren lag eindeutig auf der häufig isolierten Betrachtung von sexuellem Missbrauch an Mädchen. Dabei wurde allerdings übersehen, dass auch der sexuelle Missbrauch von Jungen, wenn auch nicht so häufig wie bei Mädchen, kein selte- nes Ereignis darstellt. Unstrittig ist sicher die Tatsache, dass es unter weiblichen Suchtmittelabhängigen ca. doppelt bis dreimal so viele Frauen mit kindlichen Missbrauchserfahrungen gibt wie bei den Männern. Unter Berücksichtigung der Tatsache, dass es eine Geschlechterverteilung von Männern zu Frauen von 3:1 im Suchthilfesystem gibt, ist die absolute Anzahl Männer mit Missbrauchserfahrungen jedoch mit denen der Frauen vergleichbar. Für Männer scheint sexueller Missbrauch darüber hinaus noch viel schamhafter besetzt zu sein, zumal er in den meisten Fällen auch als homosexueller Kontakt stattfand und bei Männern noch immer das Vorurteil besteht, dass sie unter sexuellen Übergriffen weniger leiden und für sie Sexualität grundsätzlich lustbetonter ist. Erstaunlich bleibt aber, dass es so wenige Arbeiten gibt, die sich mit den Auswirkungen sexuellen Missbrauchs bei Jungen beschäftigen. Daher scheint es sowohl für Wissenschaftler als auch für Behandler in Suchthilfeeinrichtungen unerlässlich, sich mit den eigenen Geschlechtsrollen-Klischees und jungenspezifischen Mythen auseinander zu setzen.

Missbrauch und Misshandlungen im Kindesalter stellen selten ein isoliertes Ereignis dar. Sie sind vielmehr eingebettet in ein Geflecht von weiteren negativen Kindheitserfahrungen, wie seelischer Missbrauch, Substanzkonsum der Eltern oder ein grundsätzliches Klima von Gewalt im Leben des Kindes und Heranwachsenden. Das vorherrschende Familienklima scheint dabei sozusagen den Hintergrund abzugeben, auf dem sich die einzelnen Missbrauchs- und Misshandlungserfahrungen abbilden [40, 42]. Zu wenig wird allerdings beachtet, dass es durchaus auch präventive Faktoren gibt, die Nachfolgeschäden begrenzen. Wie der Beitrag von Ullmann (2003) zeigt, verringert der Erwerb von social skills das Risiko einer Reviktimisierung [64]. Weitergehende Forschung ist nötig, um wichtige Erkenntnisse darüber zu gewinnen, warum und wie Menschen traumatische Ereignisse verarbeiten, ohne an deren Folgen zu erkranken. Implikationen sowohl für den Bereich der Prävention als auch für die therapeutische Arbeit könnten daraus abgeleitet werden [65].

Therapeutische Angebote ohne eine genaue Diagnostik im Hinblick auf Traumatisierungen und ohne ein spezielles Angebot für diese Patientengruppe verlängern unter Umständen den Leidensweg dieser Untergruppe der Substanzabhängigen. Die Behandlung von Suchtmittelabhängigen mit traumatischen Erfahrungen in ihrer Lebensgeschichte stellt eine große Herausforderung des Hilfesystems dar. Es gibt kaum adäquate therapeutische Hilfe für Patienten mit dieser Kombination von Diagnosen und nur wenige Einrichtungen halten ein spezifisches Programm für diese Patienten bereit. Dabei ist es wichtig, wie Felitti (2002) an einem Gleichnis von Rauch und Feuer ausführt [66], nicht nur die Wirkung zu bekämpfen, sondern die Ursachen zu bearbeiten.

\section{Literatur}

${ }^{1}$ Stewart SH. Alcohol Abuse in Individuals Exposed to Trauma: A Critical Review. Psychological Bulletin 1996; 120: 83-112

2 Deering CG, Glover SG, Ready D et al. Unique Patterns of Comorbidity in Posttraumatic Stress Disorder from Different Sources of Trauma. Compr Psychiatry 1996; 37: 336-346

${ }^{3}$ Brady KT. Posttraumatic Stress Disorder and Comorbidity: Recognizing the Many Faces of PTSD. J Clin Psychiatry 1997; 58: 12 - 15 
${ }^{4}$ Stewart SH, Pihl RO, Conrod PJ et al. Functional Associations among Trauma, PTSS, and Substance-Related Disorders. Addict Behav 1998; 23: $797-812$

${ }^{5}$ Breslau N. Outcomes of Posttraumatic Stress Disorder. J Clin Psychiatry 2001; 62 (suppl): $55-59$

${ }^{6}$ Breslau N. Epidemiologic Studies of Tauma, Posttraumatic Stress Disorder, and Other Psychiatric Disorders. Can J Psychiatry 2002; 47: 923-929

${ }^{7}$ Back S, Dansky BS, Coffey SF et al. Cocaine Dependence with and without Posttraumatic Stress Disorder: A Comparison of Substance Use, Trauma History and Psychiatric Comorbidity. Am J Addict 2000; 9: $51-62$

${ }^{8}$ Creamer M, Burgess P, McFarlane AC. Post-traumatic stress disorder: findings from the Australian National Survey of Mental Health and Well-being. Psychological Medicine 2001; 31: 1237- 1247

${ }^{9}$ Kessler RC, Sonnega A, Bromet E et al. Posttraumatic Stress Disorder in the National Comorbidity Survey. Arch Gen Psychiatry 1995; 52: $1048-1060$

${ }^{10}$ Breslau N, Davis GC, Andreski P et al. Traumatic Events and Posttraumatic Stress Disorder in an Urban Population of Young Adults. Arch Gen Psychiatry 1991; 48: 216 - 222

${ }^{11}$ Stein MB, Walker JR, Hazen AL et al. Full and Partial Posttraumatic Stress Disorder: Findings From a Community Survey. Am J Psychiatry 1997; 154: $1114-1119$

${ }^{12}$ Helzer JE, Robins LN, McEvoy L. Post-Traumatic Stress Disorder in the General Population. N Engl J Med 1987; 317: 1630-1634

13 Davidson JRT, Hughes S, Blazer DG et al. Post-traumatic stress disorder in the community: an epidemiological study. Psychol Med 1991; 21: $713-721$

${ }^{14}$ Kessler RC, Zhao S, Katz SJ et al. Past-Year Use of Outpatient Services for Psychiatric Problems in the National Comorbidity Survey. Am J Psychiatry 1999; 156: $115-123$

${ }^{15}$ Resnick HS, Kilpatrick DG, Dansky BS et al. Prevalence of Civilian Trauma and Posttraumatic Stress Disorder in a Representative National Sample of Women. J Consult Clin Psychol 1993; 61: 984-991

${ }^{16}$ Perkonigg A, Kessler RC, Storz S et al. Traumatic events and post-traumatic stress disorder in the community: prevalence, risk factors and comorbidity. Acta Psychiatr Scand 2000; 101: 46-59

17 Deykin EY, Buka SL. Prevalence and Risk Factors for Posttraumatic Stress Disorder Among Chemically Dependent Adolescents. Am J Psychiatry $1997 ; 154: 752-757$

18 Khantzian EJ. The self-medication hypothesis of addictive disorders. Focus on heroin and cocaine dependence. Am J Psychother 1985; 143: $1259-1264$

${ }^{19}$ Cottler LB, Compton WM, Mager D et al. Posttraumatic Stress Disorder Among Substance Users From the General Population. Am J Psychiatry 1992; 149: 664-670

20 Cottler LB, Nishith P, Compton WM. Gender Differences in Risk Factors for Trauma Exposure and Post-traumatic Stress Disorder Among Inner-City Drug Abusers In and Out of Treatment. Compr Psychiatry 2001; 42: $111-117$

${ }^{21}$ Finkelhor D, Hotaling G, Lewis IA et al. Sexual abuse in a national survey of adult men and women: Prevalence, characteristics, and risk factors. Child Abuse \& Neglect 1990; 14: 19-28

22 Bange D. Die dunkle Seite der Kindheit. Sexueller Missbrauch an Mädchen und Jungen - Hintergründe - Folgen. Volksblattverlag 1992

${ }^{23}$ MacMillan HL, Fleming JE, Trocmé N et al. Prevalence of child physical and sexual abuse in the community: results from the Ontario Health Supplement. JAMA 1997; 278: 131-135

${ }^{24}$ Richter-Appelt H. Differentielle Folgen von sexuellem Missbrauch und körperlicher Misshandlung. In: Amann G, Wipplinger R (Hrsg). Sexueller Missbrauch: Überblick zu Forschung, Beratung und Therapie. Tübingen: Dgvt-Verlag, 1997: 201 - 217

${ }^{25}$ Murphy SM, Peters FSD. Profiling child sexual abusers: psychological considerations. Criminal Justice and Behavior 1992; 19: 194-216

${ }^{26}$ Schäfer M, Schnack B, Soyka M. Sexueller und körperlicher Missbrauch während früher Kindheit und Adoleszenz bei späterer Drogenabhängigkeit. Psychother Psychosom med Psychol 2000; 50: 38-50

27 Simpson TL, Miller WR. Concomitance between childhood sexual and physical abuse and substance use problems. Clinical Psychology Review 2002; 22: $27-77$

${ }^{28}$ Haskett ME, Marziano B, Dover ER. Absence of Males in Maltreatment Research: A Survey of Recent Literature. Child Abuse Negl 1996; 20: $1175-1182$
${ }^{29}$ Burnam MA, Stein JA, Golding JM et al. Sexual Assault and Mental Disorders in a Community Population. Journal of Consulting and Clinical Psychology 1988; 56: $843-850$

${ }^{30}$ Mullen PE, Martin JL, Anderson JC et al. Childhood Sexual Abuse and Mental Health in Adult Life. Britich Journal of Psychiatry 1993; 163: $721-732$

${ }^{31}$ Wilsnack SC, Vogeltanz ND, Klassen AD et al. Childhood Sexual Abuse and Women's Substance Abuse: National Survey Findings. J Stud Alcohol 1997; 58: $264-271$

${ }^{32}$ MacMillan HL, Fleming JE, Streiner DL et al. Childhood Abuse and Lifetime Psychopathology in a Community Sample. Am J Psychiatry 2001; 158: $1878-1883$

${ }^{33}$ Kendler KS, Bulik CM, Silberg J et al. Childhood Sexual Abuse and Adult Psychiatric and Substance Use Disorders in Women. Arch Gen Psychiatry 2000; 57: 953 - 959

${ }^{34}$ Silverman AB, Reinherz HZ, Giaconia RM. The Long-Term Sequelae of Child and Adolescent Abuse: a Longitudinal Community Study. Child Abuse \& Neglect 1996; 20: 709-724

${ }^{35}$ Duncan RD, Saunders BE, Kilpatrick DG et al. Childhood Physical Assault as a Risk Factor for PTSD, Depression, and Substance Abuse: Findings From a National Survey. Am J Orthopsychiatry 1996; 66: $437-448$

${ }^{36}$ Spak L, Spal F, Allebeck P. Factors in Childhood and Youth Predicting Alcohol Dependence and Abuse in Swedish Women: Findings from a General Population Study. Alcohol \& Alcoholism 1997; 32: 267-274

37 Winfield I, George LK, Swartz M et al. Sexual Assault and Psychiatric Disorders Among a Community Sample of Women. Am J Psychiatry 1990; 147: $335-341$

38 Bulik CM, Prescott CA, Kendler KS. Features of childhood sexual abuse and the development of psychiatric and substance use disorders. British J Psychiatry 2001; 179: 444-449

${ }^{39}$ Felitti VJ, Anda RF, Nordenberg D et al. Relationship of Childhood Abuse and Household Dysfunction to Many of the Leading Causes of Death in Adults. Am J Prev Med 1998; 14: 245-258

${ }^{40}$ Dube SR, Anda RF, Felitti VJ et al. Adverse Childhood experiences and personal alcohol abuse as an adult. Addictive Behaviors 2002; 27: $713-725$

${ }^{41}$ Dube SR, Felitti VJ, Dong M et al. Childhood Abuse, Neglect, and Household Dysfunction and the Risk of Illicit Drug Use: The Adverse Childhood Experiences Study. Pediatrics 2003; 111: 564-572

${ }^{42}$ Edwards VJ, Holden GW, Felitti VJ et al. Relationship Between Multiple Forms of Childhood Maltreatment and Adult Mental Health in Community Respondents: Results From the Adverse Childhood Experiences Study. Am J Psychiatry 2003; 160: 1453-1460

${ }^{43}$ Malinosky-Rummell R, Hansen DJ. Long-Term Consequences of Childhood Physical Abuse. Psychological Bulletin 1993; 114: 68 - 79

${ }^{44}$ Hildyard KL, Wolfe DA. Child neglect: developmental issues and outcomes. Child Abuse \& Neglect 2002; 26: 679-695

45 Krausz M, Briken P. Sexueller Missbrauch bei opiatabhängigen Frauen in Relation zu biographischen Faktoren, Suchtentwicklung und psychischer Symptomatik. Suchttherapie 2002; 3: 178-183

${ }^{46}$ Quimette PC, Kimerling R, Shaw J et al. Physical and Sexual Abuse Among Women and Men with Substance Use Disorders. Alcoholism Treatment Quarterly 2000; 18: 7-17

47 Wallen J, Berman K. Possible Indicators of Childhood Sexual Abuse for Individuals in Substance Abuse Treatment. Journal of Child Sexual Abuse 1992; 1 : $63-74$

${ }^{48}$ Gil-Rivas V, Fiorentine R, Anglin MD. Sexual Abuse, Physical Abuse, and Posttraumatic Stress Disorder among Women Participating in Outpatient Drug Abuse Treatment. J Psychoactive Drugs 1996; 28: 95-102

${ }^{49}$ Clark DB, Lesnick L, Hegedus AM. Traumas and Other Adverse Life Events in Adolescents With Alcohol Abuse and Dependence. J Am Acad Child Adolensc Psychiatry 1997; 36: 1744-1751

${ }^{50}$ Clark HW, Masson CL, Delucchi KL et al. Violent traumatic events and drug abuse severity. J Subst Abuse Treat 2001; 20: 121 - 127

${ }^{51}$ Ballon BC, Courbasson CMA, Smith PD. Physical and Sexual Abuse Issues Among Youths With Substance Use Problems. Can J Psychiatry 2001; 46: 617-621

52 Langeland W, van den Brink W, Draijer N. Trauma, Trauma-Related Distress, and Perceived Parental Dysfunction: Associations with Severity of Drinking Problems in Treated Alcoholics. J Nerv Ment Dis 2002; 190: $337-340$ 
53 Thompson Fullilove M, Fullilove RE, Smith M et al. Violence, Trauma, and Post-Traumatic Stress Disorder Among Women Drug Users. J Trauma Stress 1993; 6: $533-543$

54 Brady KT, Killeen T, Saladin ME et al. Comorbid Substance Abuse and Poststraumatic Stress Disorder. Characteristics of Women in Treatment. Am J Addict 1994; 3: 160 - 164

55 Simpson TL. Women's Treatment Utilization and ist Relationship to Childhood Sexual Abuse History and Lifetime PTSD. Substance Abuse 2002; 23: $17-30$

${ }^{56}$ Simons L, Ducette J, Kirby KC et al. Childhood Trauma, Avoidance Coping, and Alcohol and Other Drug Use Among Women in Residential and Outpatient Treatment Programs. Alcoholism Treatment Quarterly 2003; 21: $37-54$

57 Triffleman EG, Marmar CR, Delucchi KL et al. Childhood Trauma and Posttraumatic Stress Disorder in Substance Abuse Inpatients. J Nerv Ment Dis 1995; 183: $172-176$

${ }^{58}$ Harrison PA, Edwall GE, Hoffman NG et al. Correlates of Sexual Abuse Among Boys in Treatment for Chemical Dependency. Journal of Adolescent Chemical Dependency 1990; 1: 53-67

59 Jarvis TJ, Copeland J, Walton L. Exploring the nature of the relationship between child sexual abuse and substance use among women. Addiction 1998; 93: 865-875
${ }^{60}$ Roy A. Childhood trauma and neutroticism as an adult: possible implication for the development of the common psychiatric disorders and suicidal behaviour. Psychol Med 2002; 32: 1471 - 1474

61 Quimette PC, Brown PJ, Najavits LM. Course and Treatment of Patients with Both Substance Use and Posttraumatic Stress Disorder. Addictive Behaviors 1998; 23: 785 - 795

62 Jacobsen LK, Southwick SM, Kosten TR. Substance Use Disorders in Patients with Posttraumatic Stress Disorder: A Review of the Literature. Am J Psychiatry 2001; 158: 1184- 1190

${ }^{63}$ Ruggiero J, Bernstein DP, Handelsman L. Traumatic Stress in Childhood and Later Personality Disorders: A Retrospective Study of Male Patients With Substance Dependence. Psychiatric Annals 1999; 29: $713-721$

64 Ullman SE. A critical review of field studies on the link of alcohol and adult sexual assault in women. Aggression and Violent Behavior 2003; 8: $471-486$

${ }^{65}$ Banyard VL. Explaining links between sexual abuse and psychological distress: identifying mediating processes. Child Abuse \& Neglect 2003; 27: 869-875

${ }^{66}$ Felitti VJ. Belastungen in der Kindheit und Gesundheit im Erwachsenenalter: die Verwandlung von Gold in Blei. Z Psychosom Med Psychother 2002; 48: 359-369 